\title{
Cloud Calculator: A cloud assessment tool for the Public Administration
}

\author{
Eduardo Cidres \\ INESC-ID, Instituto Superior Técnico, \\ Universidade de Lisboa, Lisboa, \\ Portugal \\ eduardo.cidres@tecnico.ulisboa.pt
}

\author{
André Vasconcelos \\ INESC-ID, Instituto Superior Técnico, \\ Universidade de Lisboa, Lisboa, \\ Portugal \\ andre.vasconcelos@tecnico.ulisboa.pt
}

\author{
Filipe Leitão \\ TicAPP, Agência para a Modernização \\ Administrativa, Lisboa, Portugal \\ filipe.leitao@ticapp.gov.pt
}

\begin{abstract}
As cloud computing is becoming more popular as a way to host services and to improve businesses, its adoption still remains to be a clearly defined process. Specifically, when the adoption is to be made within the public administration (where additional constraints apply when compared to the private sector). Legislation needs to be created, standards need to be developed, and public organizations need to be in synch with their cloud goals and approaches. This paper proposes a tool that supports the architecture assessment of cloud migration or adoption initiatives. A systematic literature review is conducted to gain a better understanding of the characteristics of a system that are important to consider when analyzing the cloud viability of a system. Considering the technical aspects of cloud adoption, this paper proposes several criteria linked to software qualities. With the criteria set established, weights are then defined according to their respective importance in the system, supporting the readiness level classification that a system has regarding cloud computing, based on multi-criteria decision analysis. The developed solution is then applied in a case study, assessing the usefulness and effectiveness of the proposed tool for the cloud adoption process.
\end{abstract}

\section{CCS CONCEPTS}

- Computer systems organization $\rightarrow$ Architectures; $\bullet$ Applied computing $\rightarrow$ Computers in other domains.

\section{KEYWORDS}

Cloud adoption, Migration tool, Feasibility assessment, MCDM

\section{ACM Reference Format:}

Eduardo Cidres, André Vasconcelos, and Filipe Leitão. 2020. Cloud Calculator: A cloud assessment tool for the Public Administration. In The 21st Annual International Conference on Digital Government Research (dg.o '20), fune 15-19, 2020, Seoul, Republic of Korea. ACM, New York, NY, USA, 8 pages. https://doi.org/10.1145/3396956.3396964

Permission to make digital or hard copies of all or part of this work for personal or classroom use is granted without fee provided that copies are not made or distributed for profit or commercial advantage and that copies bear this notice and the full citation on the first page. Copyrights for components of this work owned by others than ACM must be honored. Abstracting with credit is permitted. To copy otherwise, or republish, to post on servers or to redistribute to lists, requires prior specific permission and/or a fee. Request permissions from permissions@acm.org.

dg.o '20, June 15-19, 2020, Seoul, Republic of Korea

(c) 2020 Association for Computing Machinery.

ACM ISBN 978-1-4503-8791-0/20/06 . .\$15.00

https://doi.org/10.1145/3396956.3396964

\section{INTRODUCTION}

\subsection{Motivation}

In the last twenty years cloud computing has been an everincreasing topic in the research and development of new technologies that are able to leverage the digital transformation benefits. Some of these benefits are obvious, such as upfront investment cost reduction, flexibility, and agility when deploying services in the cloud. Others are not so clear, like environmental and energy efficiency or universal access, where cloud services can be accessed from anywhere and any device, supporting remote work [1].

But the cloud adoption does not come without its challenges. One of the biggest pertains to security, since it brings new vulnerabilities and threats alongside with all of the benefits it provides [2]. Another challenge refers to the software product architecture, where a loosely coupled architecture is required - which is something that does not exist in most legacy applications [3].

Despite all the efforts made, cloud migration remains a process with many uncertainties, specifically when it comes to governmental organizations since these have additional constraints when tackling the adoption of cloud computing. This is mostly due to the lack of legislation and standards on how to approach a change as important as the adoption of cloud [4]. Even when taking a look at the development of cloud frameworks, it can be observed that until today there is not a unified approach to take when facing the possibility of cloud adoption [3, 5]-[8].

Some tools were developed to aid the process of decision making in cloud computing adoption, but all are built with fixed criteria in mind having a certain lack in customization [8]-[11]. Also, none of these allow the measurement of the cloud readiness of a system, which is something very useful specially when the subject of evaluation is a legacy system. In this case, different aspects need to be assessed and considered to conclude if the changes required to adapt the system to the cloud are worth the benefits that come with the migration. These required changes pose an even greater risk for public administrations since budget constraints are usually higher and lack of legislation seriously affects the time spent on the adoption/migration process.

Technological characteristics of software such as required scalability or the amount of security measures needed, are crucial to the definition of the boundaries that allow to make a solid analysis as to support an informed choice on whether to migrate/adopt cloud or not. 


\subsection{Research Objectives}

The purpose of this research is to propose a tool that enables cloud adoption decision and respective migration in the public administration, and if possible, in more general cases. The cloud adoption decision tool is based on an existing framework considering its degree of maturity.

By performing a systematic literature review of existing migration models and frameworks, an analysis is made on the criteria utilized in each one. With that information put together, a set of criteria is proposed in order to serve as the base of the cloud adoption decision tool. Following the definition of the criteria to be considered in the research, another analysis was conducted on the existing multi-criteria decision analysis techniques that can be applied. By comparing each of the existing solutions, the technique that best suits the needs is chosen. Despite using specific criteria as the core of the work, the tool proposed supports customization and flexibility. This also allows room for future changes that may occur to the cloud environment, therefore extending the lifetime and usability of the tool.

The paper has the following structure. In section 2 the background of cloud technology and current state is provided, ranging from the challenges faced with cloud adoption to the analysis of multi-criteria decision-making techniques. In section 3 the systematic literature review is conducted as well as a brief analysis on developed cloud migration frameworks and multi-criteria decision analysis methods. The current state of cloud adoption across multiple nations' public administrations in next presented, even dedicating a section to highlight the cloud program of Portugal.

\section{BACKGROUND}

\subsection{Challenges in cloud migration}

Despite the benefits that come with the migrating to the cloud, they are only achieved if the applications are planned and developed with the cloud mindset and architecture [12].

This makes cloud transitioning challenging for companies that have legacy applications based on principles not compatible with the cloud. To make legacy applications better candidates to move to the cloud, it is usually required to rebuild the underlying structure of applications [3]. Since cloud computing requires a loosely coupled architecture, it is ideal for stateless services and applications, where there are no infrastructure dependencies. Most legacy applications rely on stateful services, and the cost of making a transition into stateless can make the transition to cloud unfeasible.

One of the major concerns and challenges presented by the cloud is security [2]. Security may be maximized if the applications are designed from the ground up with the cloud security architecture in mind.

Another challenge is the impact made in the organization when transitioning to the cloud. Managing change and the people that work inside the company is difficult and poses as a risk, although this can be mitigated by starting with smaller and lower-risk initiatives as early candidates for cloud computing projects. This allows a paced adaptation process to the new business process, by giving time to new cloud specialized workers to teach and support the ones that are making the transition to the cloud model.

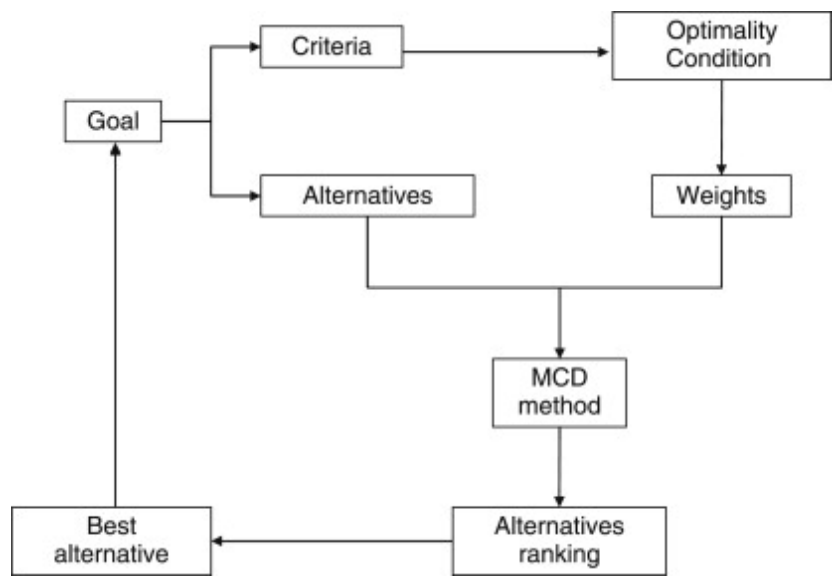

Figure 1: General structure of an MCDM process

\subsection{Multi-criteria decision making}

Multi-criteria decision making (MCDM) consists in finding the best solution from a pool of possible candidates according to selected criteria, by basing the decision on mathematical and programming tools [13]. The process of applying MCDM techniques is made of several phases such as establishing the criteria to use in the evaluation, the number of alternative solutions one wants to decide upon, applying weights to the previously defined criteria, and then apply the MCD method itself. This procedure is more comprehensible in figure 1 [14].

There are two types of MCDM approaches that can be used: there is Multi-Attribute utility Theory (MAUT) and outranking approaches [13]. MAUT consists in obtaining a function that represents the utility of an alternative, where the alternative then attributes a marginal utility, with a number representing the preferability of the alternative. The total utility score results from the sum of all marginal utilities.

On the other hand, outranking approaches are based on pairwise comparisons between the aspects of the considered solutions as to establish which solution is better than another.

Within these two approaches there are commonly used techniques such as AHP, ELECTRE, PROMETHEE, TOPSIS and VIKOR.

AHP is a technique that takes into account the mutual relationship between criteria, since it is not always possible to keep them all independent from each other in real-world problems [15]. It is based on pairwise comparisons of the criteria, where these are organized in a hierarchical relationship.

ELECTRE is a technique that falls in the class of outranking methods, which consists in performing pairwise comparisons between alternatives and creates and outranking relationship among them [15]. The created relationship is then used to identify and exclude the alternatives that are dominated by other alternatives, reducing the total number of possible alternatives.

PROMETHEE stands for preference ranking organization method of enrichment evaluation and is an outranking method just like ELECTRE, but unlike it, it stands as an improved technique over ELECTRE. The difference between the two lies in the pairwise comparison stage [15]. Instead of only verifying if an alternative is 
Table 1: Cloud adoption criteria for technology/systems

\begin{tabular}{|c|c|c|c|c|c|c|c|c|c|c|c|c|c|c|c|c|c|}
\hline \multirow[b]{2}{*}{ Criteria } & \multirow[t]{2}{*}{ Sub-Criteria } & \multicolumn{16}{|c|}{ Articles supporting criteria } \\
\hline & & [25] & [27] & [18] & [21] & [7] & [3] & [22] & [23] & [8] & [11] & [5] & [19] & [20] & {$[26]$} & [17] & [24] \\
\hline \multirow{5}{*}{ RISKS } & 1. Complexity/Interoperability & & & $\checkmark$ & & $\checkmark$ & $\checkmark$ & & & $\checkmark$ & & $\checkmark$ & $\checkmark$ & $\checkmark$ & & $\checkmark$ & \\
\hline & 2. Compatibility & & & $\checkmark$ & $\checkmark$ & & $\checkmark$ & $\checkmark$ & $\checkmark$ & & & $\checkmark$ & $\checkmark$ & $\checkmark$ & & $\checkmark$ & $\checkmark$ \\
\hline & $\begin{array}{l}\text { 3. Availability and } \\
\text { accessibility/SLA }\end{array}$ & $\checkmark$ & & $\checkmark$ & $\checkmark$ & $\checkmark$ & & & & $\checkmark$ & $\checkmark$ & $\checkmark$ & $\checkmark$ & $\checkmark$ & $\checkmark$ & & $\checkmark$ \\
\hline & 4. Security & $\checkmark$ & $\checkmark$ & $\checkmark$ & $\checkmark$ & $\checkmark$ & & & $\checkmark$ & $\checkmark$ & $\checkmark$ & $\checkmark$ & $\checkmark$ & $\checkmark$ & $\checkmark$ & & $\checkmark$ \\
\hline & 5. Portability & & & & & $\checkmark$ & & & & & & & & & & & \\
\hline \multirow{7}{*}{ QUALITIES } & 6. Scalability & & & & $\checkmark$ & $\checkmark$ & $\checkmark$ & $\checkmark$ & & $\checkmark$ & & $\checkmark$ & & $\checkmark$ & & & \\
\hline & 7. Trialability & & & $\checkmark$ & & & & & $\checkmark$ & & & & & & & $\checkmark$ & \\
\hline & 8. Testability & & & & & $\checkmark$ & $\checkmark$ & $\checkmark$ & & & & & & & & & \\
\hline & 9. Backup/Recovery & & & & $\checkmark$ & & & & & & & & & $\checkmark$ & & & \\
\hline & 10. Performance & & $\checkmark$ & & $\checkmark$ & $\checkmark$ & & & & $\checkmark$ & $\checkmark$ & & & & & & \\
\hline & 11. Elasticity & & & & $\checkmark$ & & & & & & & & & & & & \\
\hline & 12. Continuous monitoring & & & & & & $\checkmark$ & & & & & & & & & & \\
\hline
\end{tabular}

better than another, it also checks the degree to which an alternative is better or worse than the other.

TOPSIS tries to select an alternative with the following two conditions: it needs to be the closest to the ideal solution while simultaneously being the farthest from the anti-ideal solution [15].

VIKOR focuses on the ranking and selection from a set of alternatives in the existence of conflicting criteria [16]. It is similar to the TOPSIS method, the difference being in that VIKOR tries to find the alternative that is closest to the ideal solution.

\section{RELATED WORK}

\subsection{Frameworks and multi-criteria decision making}

In order to establish the criteria for assessing cloud adoption feasibility within public administration institutions, a systematic literature review of existing cloud migration frameworks and their respective utilized criteria was conducted in order to analyze what solutions and parameters are the most popular and comprehensive.

The articles used in the literature review are present in journals and conferences obtained using search engines of publishers such as Springer, IEEE, ResearchGate and Elsevier. Others were obtained through the Google Scholar search engine. It is also worth noting that only articles published starting in the year of 2015 were considered, in order to constrain the number of results further as well as having the most recent research material on cloud computing adoption.

The keywords used to conduct the search for relevant articles were a mix of the following: Cloud, Adoption, Migration, Framework, Criteria, Assessment, Tool, Feasibility, Government.

After collecting 33 possibly relevant articles using the method described above, a manual review was conducted to filter them further. The papers that present literature reviews about cloud adoption criteria and frameworks, as well as those that propose their own frameworks and decision support tools to be used ,or that extend already existing tools and frameworks were further assessed. Articles that present the aforementioned aspects from a particular country's cloud strategy point of view would have been the ideal results; however only 3 of the 33 articles found were able to fulfil these requirements. The majority of governmental cloud strategies that are publicly available do not go into the desired level of technological aspects and criteria used for the evaluation of cloud adoption/migration. This resulted in a total of 16 relevant articles that are further studied.

3.1.1 Evaluating criteria for cloud adoption. By analyzing the 13 articles that either specifically mention criteria used in the phases of cloud feasibility analysis/assessment or mention cloud migration goals that can be translated into criteria, the following table (table 1) was constructed. This was made by merging similar characteristics with similar definitions present in several articles. Some less popular criteria were also included due to its considered relevance to the work to be developed.

- The complexity of current systems was first derived from analyzing [5] (also appearing in [3, 8, 17]). This criteria allows to assess the degree of technical complexity of the current system (in the case of legacy migration) or of the new system that is to be implemented directly in the cloud. In the case of legacy migration, this can be related to the concept of interoperability present in $[3,7,18]-[20]$ which refers to the level of application integration across multiple platforms, contributing to the level of complexity present/required in the application.

- Compatibility with current systems according to [5] is the level of modularity present in the current system and the ability to seamlessly interface with other applications that may not be in the cloud and that are already in use. This concept is also mentioned in [3,17]-[24] even though in most of them it is not as well defined.

- Availability and accessibility as mentioned in [18, 25, 26] refer to their respective levels of requirement by the system and the existence of redundancy measures within it, as well as the extent up to which the cloud providers can support these requirements. It also refers to the ease of access to the 
system from different devices [21]. These two aspects are sometimes combined and used as one [5, 7]. Both characteristics are related to what the cloud providers present as SLAs (service level agreements)[24], since the requirements of availability of service and accessibility are some of the aspects covered in these agreements in order to give reassurance to CSP clients while giving them a realistic view of what level of service quality to expect.

- Security as defined in [5] represents the security measures that are necessary in the system: if they need to be location based, if the cloud provider is able to replicate the same measures such as protecting organization data and maintaining the same level of privacy and confidentiality of the data $[18,23,24,26]$. Not only this but it is also important to assess the existing level of expertise in dealing with security threats whether it is from the current IT staff or expertise given by the cloud provider. This represents the major risk in cloud adoption [20], having only two articles that do not mention it directly. The article [25] also underlines the importance in having control mechanisms able to respond to security threats and incidents, including the existence of security policies as well as compliance to rules and regulations. Although the latter mentioned are not directly part of the technology that is being considered for migration, they are fundamental aspects to consider before the appliance of a system migration.

- Portability as mentioned in [7] refers to the disruption level that system will suffer when migrated to cloud, or event between cloud providers. This criterion is related to the ease of decoupling the system from the underlying infrastructure of the cloud provider, therefore avoiding vendor lock-in.

- Scalability as defined in [5] is the ability to keep up with an increasing workload by incrementally increasing a proportionate amount of computational resources [21].

- Trialability can be portrayed as the adequacy and availability given by the cloud providers to try out their services before the actual use [17, 18, 23].

- Testability has relevance due to the advantageous merging between agile development and cloud computing. Therefore, the level of ease or improvement in deployment speed when testing and developing a system [7] before putting it fully operational in the cloud can be considered as important factor when weighting the decision of cloud adoption/migration. Although not specifically mentioned, it can be included in the agility category mentioned in [22].

- Depending on the system and organization needs, backup and recovery are important factors when opting for cloud migration as seen in [20,21].

- Performance as described in [7] is the throughput speed and the existing computational power, where its assessment consists in the validation according to the system requirements.

- Elasticity referenced in [21] is the ability to increase and decrease computational power in a simple and instantaneous manner according to the needs of the system.

- Continuous monitoring in only defined in [3] as the level of service provided when it comes to the ability to monitor the system in the cloud and cloud resources to assure SLA compliance.

3.1.2 Framework analysis. Upon reviewing the articles that proposed their own cloud migration frameworks or extended existing ones, the information related to the number of phases identified in the migration process, their names, definitions and theoretical background were compiled and compared to see if there are differences in the definitions and approaches, particularly in the technical assessment phase where this work will be focused on.

The article [25] is the only one to use a single phase to include all the work that is required before the migration phase, where the assessment part is included in the planning phase. All others have migration phases dedicated to the assessment process [8, 19, 27, 28], one even goes to the extent of splitting the assessment phase into two, as it can be seen in [8], where relevance is given to both the business and technical aspects that are required to be analyzed when considering cloud migration. There is also an article [27] that takes into consideration the existing knowledge about cloud computing within the organization, being part of the Initiation phase, as well as identifying opportunities and threats that come with the adoption of cloud. One other article [28] starts the lifecycle of adoption with the feasibility analysis stage, where it states the importance of determining if the current financial and technical aspects required are propitious to a successful cloud migration. In the article [28], the Assessment phase is used in a higher level to represent the whole evaluation process of elements required to proceed with cloud migration such as costs, strategies to be followed, operational requirements, high level needs as well as other conditions.

When it comes to the approaches used in each of the articles, there are some who are well defined $[25,27,28]$ and there are others that result in the confluence of common aspects of conducted literature reviews $[8,19]$. These present a more distanced view from singular theoretical basis, but at the same time by unifying common points from different approaches they also consolidate the knowledge base required when considering cloud computing adoption. On the other hand, by using a specific approach such as risk management [25], it also allows us to deepen the knowledge of ramifications that cloud computing adoption possesses as well as its consequences. In order to best manage these consequences, the corresponding controls are defined for each of the four dimensions of sustainability also identified in the article. Another article [27] resorts to a meta-synthesis approach to perform literature review of previously published studies, so as to suggest a new comprehensive cloud migration framework. It then presents the new framework that consists in seven categories and fifteen sub-categories. Adding to that, a maturity model (ClM3) is also combined to aid the various framework migration phases. One other article has its approach based on the brokerage model [28], with the aim of automating both the cloud service selection and deployment phases in the cloud migration process. With this approach, a phase of the migration process is entirely dedicated to brokerage, where an economic model is used to aid organizations to choose the best cloud provider when taking into consideration the requirements of the organizations.

After taking all the analyzed frameworks into consideration the one that presents the most extensive and complete assessment phase 
is [27], which better aligns with the work to be developed in this research. By covering all the aspects to take into consideration when assessing the cloud migration/adoption feasibility of a solution, it serves as a solid base, upon which will be used to develop the tool.

\subsection{Current state of cloud in the Portuguese public administration}

The purpose of cloud adoption in the Portuguese public administration is to improve the quality of public services provided while achieving reduced costs for both citizens and companies and reducing public expenditure, particularly in Information and Communication Technologies (ICT) [1].

The Portuguese government identified two strategic sectors that focus precisely in cost reductions and in the implementation of common IT solutions across the public administration. An initiative was launched in order to rationalize the already existing data centers with the intent to fulfill the following objectives which are explained next. First, there is the increase the profitability of the investments made in public infrastructure and data centers, resorting to the private cloud whenever it proves to be a more competitive solution. Another objective is the unification of existing data centers. Adding to this is providing guidance in order to promote the adoption of cloud computing in the public administration. Ultimately, there is the assurance of the adoption of more agile and transparent solutions, as well as its management, with improved security, lower costs and efficient use of resources.

The results of the initiative showed the fragmentation of acquisition and management of infrastructure, with a lot of redundant and replicated systems. It was also noted that the use of open source software and norms was lacking, and cloud adoption benefits were identified.

\subsection{Cloud adoption in public administrations}

Other countries have already made great progress in the cloud adoption for their respective governments and public administration. Each one with a slightly different approach since the existing conditions are specific to each country such as legislation, geopolitical status, previous built infrastructure, willfulness to change, among others. Additional information of each of the countries' cloud adoption drivers and objectives can be observed in table 2

When it comes to Australia, particularly Western Australia, a certain emphasis was made on the reasons to adopt a public cloud model [29]. Some of these are disaster recovery, support of special events requiring large capabilities of infrastructure and the cheaper upfront cost of the development of proofs of concept.

It is also worth noting that the Australian government opted to keep the control and responsibility of ICT delivery agency independent, favoring a more decentralized approach to ICT in the public administration.

In the USA the development of a roadmap for cloud adoption among governmental agencies was carried out by NIST [30], covering all topics ranging from the establishment of priority requirements that need to be satisfied in order to transition further into cloud adoption, to the definition of standards, guidelines and technology required for portability, security and interoperability. This served as a basis for further development in both the implementation of cloud solutions in institutions and maturity of models and adopted frameworks.

The UK made it clear in its Cloud First policy to prioritize the adoption of public cloud solutions rather than other cloud deployment models [31]. Although there are situations where other deployment models would be more appropriate, the UK government believes that the main benefits of cloud adoption are achieved with the use of a public cloud model.

Italy's process of cloud adoption in its public administration started with the merging and consolidation of existing governmentowned data centers [32], a similar situation to the one of Portugal. After this process was concluded, it allowed for the use of cloud services whether they relied on the governmental cloud for services that manipulate more sensitive data or other non-critical data applications hosted on third-party cloud providers.

Spain's cloud adoption is interesting due to the already existing communication line infrastructure Red SARA (the Telecommunication Network of the Spanish Public Administration), that provided interconnectivity among public administration entities as well as connectivity with EU institutions [4].

\section{CLOUD CALCULATOR ARCHITECTURE}

In order to address the problem of cloud adoption/migration decision of a system this section proposes a solution. This solution comes with several challenges.

The challenges when deciding on migrating a system to the cloud are many. One of the challenges relates to selecting the right criteria to be applied to the system architecture in order to extensively measure its needs and requirements on various levels depending on the function of the system. After this, there is the problem of properly quantifying and evaluating these criteria, followed by the issue of balancing the weight that each criterion has.

The proposed solution is based in the implementation of the assessment tool as a web-based application, by using the Django web development framework for Python. A graphical representation of the solution development lifecycle is presented below by figure 2, allowing for a better view of the solution.

The core of the solution is the criteria gathered in section 3.1.1, as these better represent the aspects that need to be taken into consideration when assessing the cloud feasibility of a system/software application (table 1).

But before the implementation itself, it is vital to find the best way to quantify the weights of each of the established criteria, specifically the definition of the scale to be used and the respective meaning of both boundaries. This is done with the formulation of guide questions that allow a better understanding of what each of the possible values in the scale mean within the context of the criteria.

Consequently, fine tuning of the criteria is also needed to be made in order for these to be as clear as possible. With this done, the next step is choosing the best suited multi-criteria decision analysis technique to use in our tool in order to obtain the level of readiness to cloud of the evaluated system. Since MCDM is based on the ponderation of multiple possible solutions, two possibilities 
Table 2: Cloud adoption drivers and objectives in other countries

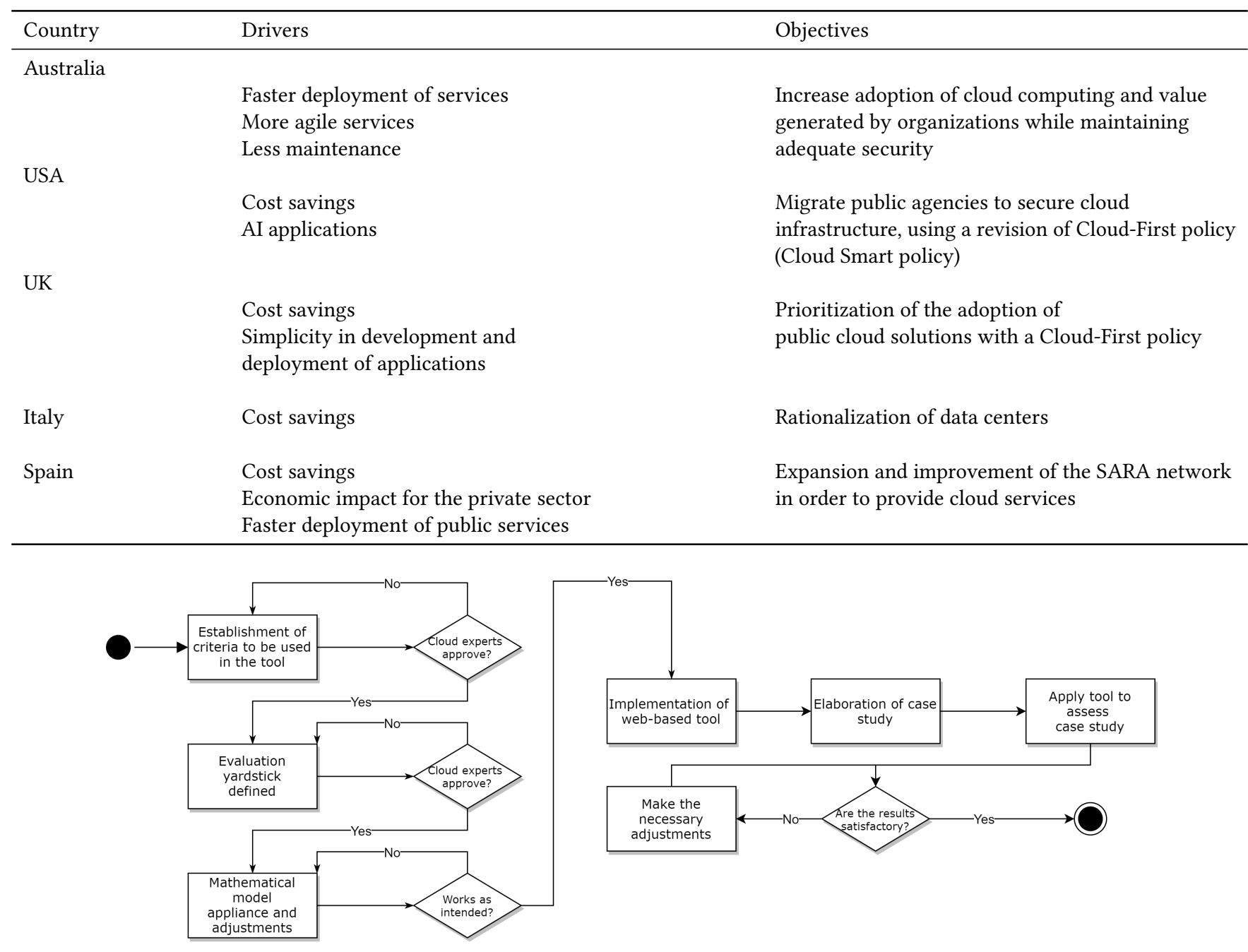

Figure 2: Tool development lifecycle

can be considered: one where cloud migration is the best solution and another one where it is best not to migrate such solution.

\section{CLOUD CALCULATOR EXAMPLE}

The following example describes the use of the tool developed. For the sake of simplicity, we will consider a database system as the subject of the cloud migration decision. Taking this into account, and assuming we would only need to base the decision on two criteria (for example scalability and security), we can start to interact with the cloud calculator tool.

In this case we are evaluating two possible alternatives/outcomes: migrate the database to the cloud or keep it hosted on-site. Before any calculations we need to establish the weights of the criteria, defining which are to take priority on the impact of the decision. This is done by assigning values between the numbers 0 and 100 to each criterion, and that are split across the used criteria set. In this case, we assume that security would take priority and would be attributed the weight of 70 , meaning that scalability would have the weight value of 30 . Since both security and scalability can be hard to quantify in an exact manner, the tool depends on ordinal qualitative scales for the scoring of each alternative for each criterion. The meanings of the scale values are provided to the user, for example where the minimal value of 1 means that the alternative presents a very poor level of scalability/security measures when compared to the other alternatives. The scale value 5 would mean that the level of scalability/security is adequate or acceptable, and the value 9 would mean that the alternatives provides the highest level of scalability/security. When looking at scalability, since the cloud is able to leverage near the maximum, a score of 9 is used. On the other hand, the on-site alternative would be heavily conditioned by regulation and time barriers, with a 3 score. As for security, and assuming that the data stored in the database is non-critical to 
Table 3: Database example application of tool

\begin{tabular}{llll}
\hline Criteria & Weights & Alternatives & Cloud hosting \\
\cline { 3 - 4 } Scalability & & On-site hosting & 9 \\
Security & 30 & 3 & 5 \\
Recommendation score & 70 & 8 & 6 \\
\hline
\end{tabular}

the point that a cloud migration can be considered, it can still be advantageous to be in control of all security mechanisms required in the database operationality. By having it hosted on-site gives us an additional layer of security, having a score of 8 . By hosting the database in the cloud, we are giving part of the security mechanisms to the responsibility of the cloud provider which can pose some risk - scoring 5.

With all required parameters defined, the multi-criteria decision making is applied, and a value of recommendation is calculated for each of the alternatives. This value of recommendation for each of the possibilities measured is then used as a base for discussion and confirmation by the cloud experts. This can be done with multiple iterations as to take advantage of additional information and knowledge obtained from previous results and respective discussions among the decision makers.

\section{FUTURE WORK}

The future work of this research consists in the creation of a case study that will be submitted to the tool with criteria weights defined with the support of experts in the field of cloud computing. The results presented by the tool will give the readiness level of cloud adoption/migration of the evaluated product/software. This will then be reviewed by experts as well, in order to obtain feedback and compare differences between the tool results and the personal analysis of the experts concerning the case study. Additionally, projects that were already implemented in the cloud in the Portuguese public administration will be used to compare with the results of the tool when applying the conditions on which they were implemented, as to have a more practical comparing method.

\section{CONCLUSION}

This paper addresses the importance of cloud adoption decision as well as the criteria to take into consideration when assessing the feasibility of cloud migration of a system. A systematic literature review is conducted to find the most popular and supported criteria for technology, as well as some considered important despite being less popular among research. The criteria are focused on the technological part of migration and is composed of 12 aspects. These are used as the basis of the evaluation of the system's cloud feasibility.

An overview of multi-criteria decision analysis techniques is provided as well as the reasons to choose a technique above others in certain situations. The architecture of the solution is provided and described, as well as the context of the work to do within the Portuguese public administration. The architecture starts with the presentation of the use cases of the tool, and then the development lifecycle of the tool is explained. An example of the utilization of the tool is given, ensuring a clear understanding of its usability and purpose . This paper contributes to address the challenges that come when performing a cloud feasibility analysis of a system, including the set of criteria used, the way that are evaluated and applied in the decision-making analysis process.

The proposed solution is a web-based tool using the criteria described in the literature review combined with a multi-criteria decision analysis in order to assess the level of cloud-readiness of a system.

\section{ACKNOWLEDGMENTS}

This work was supported by national funds through Fundação para a Ciência e a Tecnologia (FCT) with reference UIDB/50021/2020 and by the European Commission program H2020 under the grant agreement 822404 (project QualiChain).

\section{REFERENCES}

[1] "Sumário da Estratégia de Racionalização dos centros de dados e computação em nuvem na Administração Pública," 2012. [Online]. Available: https://www.ama.gov.pt/documents/24077/29210/Estrategia_Racionalizacao_ Comunicacoes.pdf/001b4c63-f24a-4462-a9f4-1ff457082252. [Accessed: 09-Oct2019].

[2] T. Halabi and M. Bellaiche, "Evaluation and selection of Cloud security services based on Multi-Criteria Analysis MCA," 2017 Int. Conf. Comput. Netw. Commun. ICNC 2017, pp. 706-710, 2017.

[3] M. F. Gholami, F. Daneshgar, G. Low, and G. Beydoun, "Cloud migration processA survey, evaluation framework, and open challenges," f. Syst. Softw., vol. 120, pp. 31-69, 2016.

[4] A.-M. Zaharia-Rădulescu and I. Radu, "Cloud computing and public administration: approaches in several European countries," Proc. Int. Conf. Bus. Excell., vol. 11, no. 1, pp. 739-749, 2017.

[5] D. Ray, "Cloud Adoption Decisions: Benefitting from an Integrated Perspective," Electron. 7. Inf. Syst. Eval., vol. 19, no. 1, pp. 3-21, 2016.

[6] M. M. Rajaeian, A. Cater-Steel, and M. Lane, "A systematic literature review and critical assessment of model-driven decision support for IT outsourcing," Decis. Support Syst., vol. 102, pp. 42-56, 2017.

[7] M. Fahmideh and G. Beydoun, "Reusing empirical knowledge during cloud computing adoption," f. Syst. Softw., vol. 138, pp. 124-157, 2018.

[8] R. Rai, G. Sahoo, and S. Mehfuz, "Exploring the factors influencing the cloud computing adoption: a systematic study on cloud migration," Springerplus, vol. 4, no. 1 , pp. $1-12,2015$

[9] A. Khajeh-Hosseini, I. Sommerville, J. Bogaerts, and P. Teregowda, "Decision support tools for cloud migration in the enterprise," Proc. - 2011 IEEE 4th Int. Conf. Cloud Comput. CLOUD 2011, pp. 541-548, 2011.

[10] R. Chard et al., "An Automated Tool Profiling Service for the Cloud," 2016 16th IEEE/ACM Int. Symp. Clust. Cloud Grid Comput., pp. 223-232, 2016.

[11] K. Ramchand, M. B. Chhetri, and R. Kowalczyk, "Towards a Flexible Cloud Architectural Decision Framework for Diverse Application Architectures," Australas. Conf. Inf. Syst., no. December, pp. 1-11, 2017.

[12] A. Mazrekaj, I. Shabani, and B. Sejdiu, "Pricing Schemes in Cloud Computing: An Overview," Int. 7. Adv. Comput. Sci. Appl., vol. 7, no. 2, pp. 80-86, 2016.

[13] M. Abdel-Basset, M. Mohamed, and V. Chang, "NMCDA: A framework for evaluating cloud computing services," Futur. Gener. Comput. Syst., vol. 86, pp. 12-29, 2018.

[14] S. Brown, Comprehensive Chemometrics: Chemical and Biochemical Data Analysis. Elsevier, 2009.

[15] Z. U. Rehman, O. K. Hussain, and F. K. Hussain, "Iaas cloud selection using MCDM methods," Proc. - 9th IEEE Int. Conf. E-bus. Eng. ICEBE 2012, pp. 246-251, 2012.

[16] S. Opricovic and G. H. Tzeng, "Compromise solution by MCDM methods: A comparative analysis of VIKOR and TOPSIS," Eur. f. Oper. Res., vol. 156, no. 2, pp. 
445-455, 2004.

[17] W. Wang, Y. Liu, Y. Liang, and K. He, "The Influential Factors of Organization Adoption of E-government Cloud," no. Fmess, pp. 283-288, 2017.

[18] A. Alkhalil, R. Sahandi, and D. John, "An exploration of the determinants for decision to migrate existing resources to cloud computing using an integrated TOE- DOI model," 7. Cloud Comput., 2017.

[19] A. Hassanzadeh and A. Moeini, "The Journal of Systems and Software A comprehensive framework for cloud computing migration using Meta-synthesis approach," vol. 128, pp. 87-105, 2017.

[20] A. Mahajani, V. Pandya, I. Maria, and D. Sharma, Ambient Communications and Computer Systems, vol. 904. Springer Singapore, 2019.

[21] A. Christoforou and A. S. Andreou, "A Multilayer Fuzzy Cognitive Maps Approach to the Cloud Adoption Decision Support Problem," 2015 IEEE Int. Conf. Fuzzy Syst., pp. 1-8.

[22] S. Khan, M. Shahid, A. Khan, and C. S. Kumar, "Multi-criteria Decision in the Adoption of Cloud Computing Services for SME 's based on BOCR Analysis," Asian f. Manag. Res., vol. 5, no. 4, pp. 621-634, 2015.

[23] F. Mohammed and O. Ibrahim, "Cloud computing adoption model for egovernment implementation," vol. 33, no. 3, pp. 303-323, 2017.

[24] Y. Liang, G. Qi, K. Wei, and J. Chen, "Exploring the determinant and influence mechanism of e-Government cloud adoption in government agencies in China, Gov. Inf. Q., vol. 34, no. 3, pp. 481-495, 2017.
[25] A. Abdul Rahman, S. Islam, C. Kalloniatis, and S. Gritzalis, "A Risk Management Approach for a Sustainable Cloud Migration," f. Risk Financ. Manag., vol. 10, no. 4, p. $20,2017$.

[26] M. O. Alassafi, A. Alharthi, R. J. Walters, and G. B. Wills, "A framework for critical security factors that influence the decision of cloud adoption by Saudi government agencies," Telemat. Informatics, vol. 34, no. 7, pp. 996-1010, 2017.

[27] N. Ahmad, "Strategy and procedures for Migration to the Cloud Computing," 2018 IEEE 5th Int. Conf. Eng. Technol. Appl. Sci., pp. 1-5, 2018.

[28] H. Malouche, Y. Ben Halima, and H. Ben Ghezala, "Enterprise Information System Migration To The Cloud: Assessment Phase," 2016 IEEE/ACS 13th Int. Conf. Comput. Syst. Appl., pp. 1-6, 2016.

[29] "Digital WA: information and communications technology (ICT) strategy 20162020." [Online]. Available: https://www.wa.gov.au/sites/default/files/2018-06/ Digital WA State ICT Strategy.pdf. [Accessed: 23-Oct-2019].

[30] L. Badger, D. Bernstein, R. Bohn, F. De Vaulx, and M. Hogan, "Special Publication 500-293 US Government Cloud Computing Technology Roadmap Volume I HighPriority Requirements to Further USG Agency Cloud," vol. I.

[31] "Government cloud first policy," 2017. [Online]. Available: https://www.gov.uk/ guidance/government-cloud-first-policy\#public-cloud-first. [Accessed: 25-Oct2019].

[32] "Three-Year Plan for ICT," 2019. [Online]. Available: https://docs.italia.it/media/ pdf/pianotriennale-ict-doc-en/stabile/pianotriennale-ict-doc-en.pdf. [Accessed: 23-Oct-2019]. 